\title{
遥感航测技术在地图测绘中的应用探析
}

\author{
刘雨川 \\ 辽宁省自然资源事务服务中心，辽宁 沈阳 110032
}

\begin{abstract}
[摘要] 近代科学技术的迅速变革、创新和普及, 推动了所有部门的高质量的发展和进步, 许多有关的先进技术已被纳入测绘 工作, 这极大地提高了测绘工作的整体效率和质量, 为保证最终地图的有效性、可靠性、精确性, 各种制图工作都必须采用 最新的制图方法, 有效提高制图产品的总体质量, 并有效避免各种问题的不利影响和负面的干扰, 进一步的提高制图的准确 性、可靠性, 避免或减少错误的发生。随着各种各样的先进技术的有效使用, 传统的绘图已经变得更有效率、更加可靠, 更 不容易受其他因素的影响。这样就可以很容易的进行室外的测绘工作了。
\end{abstract}

[关键词] 遥感航测技术; 地图测绘; 应用

DOI: $10.33142 /$ aem.v2i9.3018 中图分类号：P237 文献标识码：A

\section{Application of Remote Sensing Aerial Survey Technology in Map Surveying and Mapping}

LIU Yuchuan

Natural Resources Affairs Service Center of Liaoning Province, Shenyang, Liaoning, 110032, China

\begin{abstract}
The rapid change, innovation and popularization of modern science and technology have promoted the high-quality development and progress of all departments. Many related advanced technologies have been incorporated into the surveying and mapping work, which greatly improves the overall efficiency and quality of the surveying and mapping work. In order to ensure the effectiveness, reliability and accuracy of the final map, all kinds of mapping work must adopt the latest mapping methods, effectively improve the overall quality of mapping products, and effectively avoid the adverse effects and negative interference of various problems, further improve the accuracy and reliability of mapping, avoid or reduce the occurrence of errors. With the effective use of various advanced technologies, the traditional drawing has become more efficient, more reliable and less susceptible to other factors. In this way, it is easy to carry out outdoor mapping work.
\end{abstract}

Keywords: remote sensing aerial survey technology; map surveying and mapping; application

引言

当前, 制图技术在不断地发展、创新, 先进的技术的有效应用显著的提高了遥感技术的发展创新的水平, 在测绘 领域广泛应用中起到了重要作用, 而当前最重要的问题是如何更加有效地利用遥感技术, 推进测绘行业的高质量发展。 随着科技水平的不断提高, 我国的测绘技术也得到了长足的发展。尤其是在遥感和航空测量技术的产生和应用方面, 地图测绘得到了很大的促进和推动, 有效提高了制图工作的整体水平, 并不断优化其应用, 对各学科的影响也在日益 的加深。需要对具体的技术应用进行深入研究。

\section{1 遥感航测技术的基本内容}

\section{1 航空摄影测图技术}

随着测绘工作的进展, 航空摄影技术得到了快速发展。具体而言, 改进和创新摄影和制图方法可以使航空摄影的效 率和质量最大化, 从而促进实时勘测工作的高质量、稳定的进行。进行航空摄影的测量工作者应具备较高的专业技术能 力和实际测绘工作中的测量操作经验, 经过了稳定可靠、标准的测绘作业之后, 随后将获得的资料和数据进行精确的分 析和计算 ${ }^{[1]}$ 。与此同时相关工作人员还需要根据已获得的图形计算结果, 测量存在问题和重要的数据, 以保证其测量工作 的准确性。另外环境因素对测量过程中信息获取的不利影响也可在一定程度上得到规避。航空摄影测量技术的充分应用, 可有效降低测量工作的难度, 进一步的提高测量的可靠性、准确性。另外, 由于复杂的环境和恶劣的天气条件, 测绘工 作往往会受到一定程度的干扰，使用这个技术就可以避免这种干扰并可大大提高测量结果的准确性、可靠性 ${ }^{[2]}$ 。

\section{2 航空测量遥感技术}

把遥感航空摄影技术与遥感技术进行深入的、高水平的结合是很有必要的, 这样可以进一步的提高测绘工作的效 率和准确性。具体来说, 遥感书的应用充分的保证了绘图整体水平和工作效率。伴随着遥感技术的不断创新、应用和 普及, 将航空摄影与遥感技术进行了充分的结合, 将推动其新的融合技术在世界各国的测绘工作中的深度应用与发展。 举例来说, 在工程项目开发以及地质勘探的过程中, 使用该技术可以有效、快速地测量地形和环境, 并确保测量人员 的安全，与此同时还大大提高了图像测量的精度、减少了现实环境、气候因素的干扰 ${ }^{[3]}$ 。 


\section{2 遥感航测技术在地图测绘应用的必要性}

遥感航测是一种通过辐射到有关物体表面机进行探测的技术类型, 以有效的探测物体的形状并且在遥感仪器中使 用感应器和反射器准确的获得测绘数据。在国民经济快速发展的今天, 测绘的作用越来越明显, 为了进一步的保证测 绘工作的科学发展, 一方面要测量准确、可靠的地理信息, 另一方面要积极主动的采用精确的、先进的、高质量的测 绘方法。应在当前的基数背景下鼓励利用遥感和航空测量技术绘制地图, 这将进一步的提高绘图的总体准确性和质量。 充分利用综合的技术，通过航空摄影探测，对测绘区域进行深度的探测和分析，制作测绘区域的照片并将图像储存在 设备服务器上, 通过随后的系统内的计算程序, 研究获取航摄照片的数据, 并对分析到的数据进行分析对遥感数据进 行分类, 向服务器管理系统的输入相关信息那么就可以产生一个立体的数据模型, 使观察者能够清楚地看到数据探测 的视觉图像。在进行绘图和修改完善的过程中, 充分利用数据可大大减少与天气以及各类影响因素有关的错误, 避免 提供绘图信息时出现的偏差和问题, 并可有效提高空中测绘工作的质量和效率, 进一步的提高成本效益, 增强测绘工 作的准确性和效率，因此，必须充分利用现有技术资源，利用遥感技术准确的绘制地图 ${ }^{[4]}$ 。

\section{3 遥感航测技术在地图测绘中的具体应用}

\section{1 对动态监测的应用}

动态监测是遥感技术最重要的组成部分, 使人们能够获取地理信息; 主要是通过远程控制空间设施和在地籍系统 中记录最新资料, 在相关部门的决策中可以提供重要投入, 而在绘图方面则可以提供信息; 通过遥感获取的数据是不 定期的, 需要加以利用。另外, 可明确界定测量区域内土地资源分布情况, 通过对 “区域” 内地球状况的比较和灾害 风险的确定, 可以判断不同时期的地壳活动。遥感使清单信息能够以数据的形式显示, 与传统的制图方法不同, 地形 测量员无需进行第二次处理, 仅需完成对现有数据的分析, 以便有效地减少制图工作, 确保其利用数字数据的质量和 效率, 例如在进行核实土地使用的数据分析过程中, 城市管理者可更准确地确定土地使用的状况, 帮助制图人员更高 效的完成这些工作测绘的任务。

\section{2 建设用地勘测界定对 GPSRTK 的应用}

GPSRTK 技术主要运用 GPS 定位对地物的相关参数进行直接的测量, 该方法相对于传统的测绘方法而言, 在测量效 率和精度方面更为突出, 且对环境要求不高, 任何气候环境都可以保证测量精度, 此外, 该技术还可实现点对点的测 量, 无需进行数据的后处理, 这使得 GPSRTK 技术无法测量的面积, 可以用网络 RTK 技术的有效应用来代替, 仅有网络 RTK 技术的有效应用, 难以完成测量任务, 特别是对于大型测绘项目而言, 该技术是非常适用的 ${ }^{[5]}$ 。

\section{3 正射影像图的制作}

我们所说的数字正射影像, 实际上是指按照地图对应的范围要求, 通过数字拼接、差分校正、剪切而形成的影像。 该图象具有地图几何精度和图象特征。该方法提取并生成的信息可用于地图更新, 可有效地提取社会经济发展中新旧 自然资源信息等, 为防灾和公共设施规划建设提供相关数据参考。其特点是精度非常的高, 信息包含的十分丰富, 速 度也很快, 同时具有直观的图示。使用该技术所实现的局部开发与放大, 能充分发挥出判别性能、测量性能和管理性 能, 因而在测绘和制图的工作中被广泛的应用。

\section{4 地形图的制作与更新}

目前社会较为关注的数字地球, 实际就是将空间数据以及信息高速公路作为基础来实现的, 数字地球的形成与发 展, 需要对空间数据基础设施以及信息高速公路进行大力的发展, 而遥感航测技术能够使空间基础数据方面的更新速 度得到有效的提升, 在对地形图进行更新时, 既可以从航天遥感获取数据, 也可以从航空摄影加以获取, 而遥感航测 技术的应用实现了新途径的有效开辟，使得快速成图以及地形图修测不再被国家和地区所限制。

\section{4 结语}

随着计算机技术、信息技术和相关测绘技术的不断创新和发展，遥感和航空测量方面都取得了十分大跨越的进步。 将遥感技术应用到当前的测绘制图过程中, 可以有效地改变传统的测绘制图方式, 进一步的提高测绘制图工作的质量 和效率, 克服测量工作中存在的困难和问题。要不断改进和完善遥感技术, 使之更好地应用于航空摄影, 进一步的提 高测量精度更好地发挥作用。另外, 在绘图过程中只有根据有关规定和标准进行测量, 才能保证图样的准确性, 提高 地图所载信息的可靠性。

\section{[参考文献]}

[1]丁波.遥感航测技术在地图测绘中的应用研究 [J].居舍,2019(33): 58-69.

[2]祖琪,罗正.遥感航测技术在地图测绘中的应用探讨 [J]. 智能城市, 2019, 5(17): 89-90.

[3] 葛珍. 遥感航测技术在地图测绘中的应用探讨 [J]. 科技创新与应用, 2019 (19) : 180-181.

[4]崔晓强. 遥感航测技术在地图测绘中的应用 [J]. 工程技术研究, 2019, 4 (8) : 93-94.

[5]刘青山。遥感航测技术在地图测绘中的应用研究 [J]. 价值工程, 2018, 37 (14) : 196-197.

作者简介: 刘雨川 (1974.9-), 大学本科, 测绘高级工程师。 